\title{
Coexistence of Parry-Romberg syndrome with homolateral segmental vitiligo
}

\author{
Marta Janowska, Katarzyna Podolec, Sylwia Lipko-Godlewska, Anna Wojas-Pelc
}

Department of Dermatology, Jagiellonian University Medical College, Krakow, Poland

Head of Department: Prof. Anna Wojas-Pelc MD, PhD

Postep Derm Alergol 2013; XXX, 6: 409-411

DOI: 10.5114/pdia.2013.39441

\begin{abstract}
Parry-Romberg syndrome or progressive facial hemiatrophy was first described by Caleb Parry in 1825 and Moritz Romberg in 1846. This disorder is characterized by slowly progressing acquired unilateral hemifacial atrophy, which affects subcutaneous tissue together with the muscles and underlying bones. The pathogenesis and precise incidence of the syndrome remain unclear. Immune-mediated processes and disturbed central regulation, leading to the hyperactivity of the sympathetic nervous system, are primarily considered in the pathogenesis of this disorder. Parry-Romberg syndrome and localized scleroderma are considered to be interrelated as both of them have a similar clinicopathological appearance. We report the case of a 46-year-old man affected by both progressive atrophy of the left side of the face and homolateral, segmental vitiligo in the left side of the trunk and face.
\end{abstract}

Key words: Parry-Romberg syndrome, facial hemiatrophy, homolateral segmental vitiligo.

\section{Introduction}

Parry-Romberg syndrome (PRS) or progressive facial hemiatrophy was first described by Caleb Parry in 1825 and Moritz Romberg in 1846 [1, 2]. This disorder is characterized by slowly progressing acquired unilateral hemifacial atrophy, which affects subcutaneous tissue together with the muscles and underlying bones. The pathogenesis and precise incidence of the syndrome remain unclear due to its rarity. Immune-mediated processes and disturbed central regulation, leading to the hyperactivity of the sympathetic nervous system, are primarily considered in the pathogenesis of this disorder [3]. Parry-Romberg syndrome and localized scleroderma are considered to be interrelated as both of them have a similar clinicopathological appearance $[1,4]$. Also, trauma, viral infections, endocrine disorders, and alterations in brain lipid metabolism are believed to be associated with this disease. Progressive facial hemiatrophy is often described as coexisting with headache episodes, epilepsy (Jacksonian crises), and trigeminal neuralgia [2]. Ocular involvement in PRS is also often mentioned [5]. In rare cases, coexistence with contralateral Poland syndrome was described [6].
Acquired homolateral segmental vitiligo is described as localized amelanotic patches in dermatomal distribution, which do not cross the midline of the body. The disease is characterized by early onset and rapid stabilization, and its pathogenesis is still unclear [7-10]. Neuronal mechanisms, somatic mosaicism, and microvascular skin homing are proposed as mechanisms underlying segmental vitiligo [8].

\section{Case report}

A 46-year-old Caucasian man was admitted to the Dermatology Department at the University Hospital in Cracow complaining of vitiligo and hemifacial atrophy. The patient reported the first appearance of depigmentation of the skin, involving the left upper limb and the left side of the trunk, at the age of 32 years. Lesions progressed, eventually affecting also the skin of the left side of the chin and neck. The lesions stabilized within a period of 6 months and it was noted that the depigmented area has not changed since then. At the age of 43, the patient first observed progressing atrophy of the left cheek with concomitant pain of the left jaw, ear, and eye. No history of trauma or injury to these sites was elicited

Address for correspondence: Marta Janowska MD, Department of Dermatology, Jagiellonian University Medical College, 8 Skawinska St, 31-066 Krakow, Poland, phone: +48 508188 942, e-mail: martawolek@o2.pl Received: 31.01.2013, accepted: 23.06.2013. 


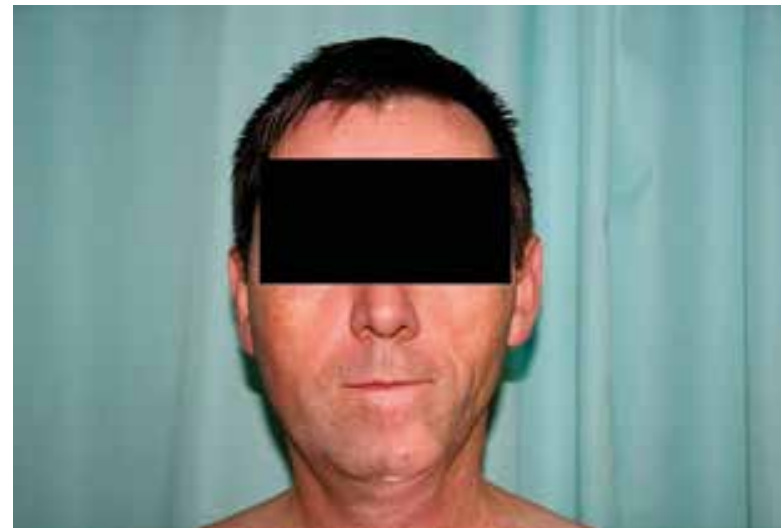

Figure 1. Facial hemiatrophy and vitiligo of the chin

at the time of admitting the patient to the Department of Neurology. The patient underwent multiple diagnostic procedures; the results were within normal ranges. Magnetic resonance imaging of the head did not reveal any focal lesions or signs of neurovascular incidents. Based on the clinical picture, trigeminal neuralgia was diagnosed and treatment with carbamazepine $200 \mathrm{mg}$ D.T.S. was prescribed. Physical examination performed on admission to the Department of Dermatology revealed asymmetry of the face with visible left facial hemiatrophy including skin and subcutaneous tissue. The amelanotic lesions had a polydermatomal distribution (C2-T9), covering the left side of the trunk, upper left limb, and left side of the chin (Figures 1 and 2). Laboratory studies, including complete blood count, erythrocyte sedimentation rate, $\mathrm{C}$-reactive protein, blood chemistry, thyroid function tests, complement components C3, C4, C1q inhibitor, anti-B2-glicoprotein antibodies, anti-Borrelia burgdorferi IgM and IgG antibodies, anti TPO, anti TG antibodies, lupus anticoagulant, and urine analysis, were all negative or within normal ranges. Autoantibody detection using indirect immunofluorescence on HEp-2 cells revealed antinuclear antibodies with granular staining pattern, titer $1: 640$, and ANA with cytoplasmic $1: 320$ and mitotic spindle staining pattern $1: 160$ (normal limit < $1: 160$ ). Double immunodiffusion and immunoblot assays were negative. Standardized ELISA tests revealed increased plasma levels of IgG anti-cardiolipin antibodies: 42.85 GPL (N: 0-10) with a negative IgM level. The results were confirmed after 6 weeks: 33.72 GPL (N: 0-10). X-ray of the cervical spine revealed no abnormalities.

\section{Discussion}

Parry-Romberg syndrome and acquired homolateral segmental vitiligo are two distinct disease entities of unknown etiology characterized by an onset in children and young adults, rapid progression followed by a subsequent long period of stabilization, and dermatomal

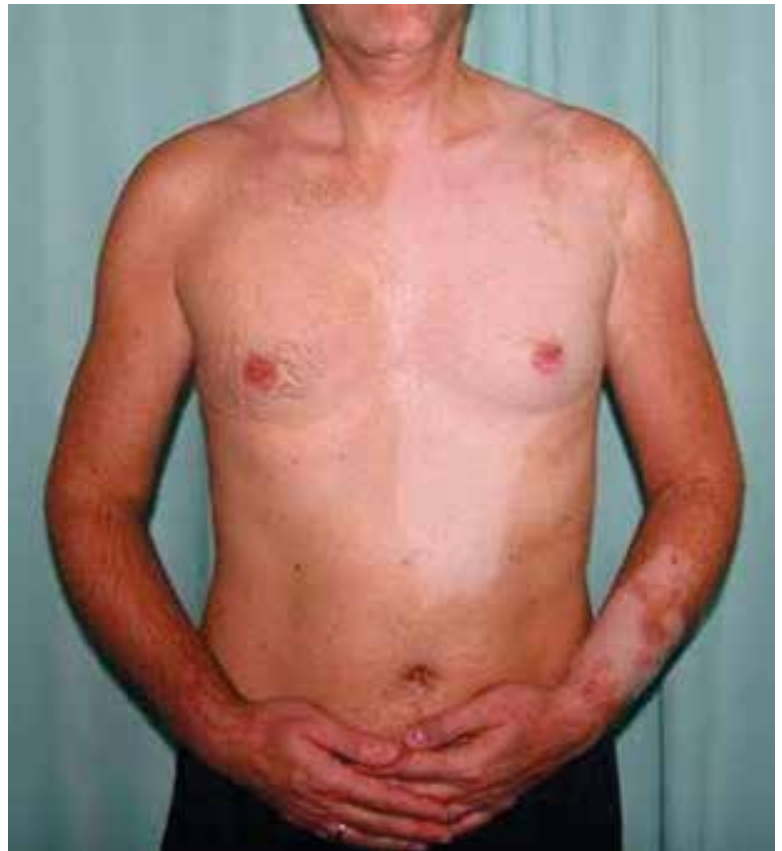

Figure 2. Vitiligo of the left upper limb and the left side of the trunk

distribution [1, 2, 4, 11]. Acquired homolateral segmental vitiligo is described as localized amelanotic patches in dermatomal distribution, which do not cross the midline of the body. The lesions usually develop between the ages of 10 and 30 years. Most commonly, segmental vitiligo involves the face, trunk, neck, extremities, and scalp in the descending order of frequency. The pathogenesis of the disease remains unclear. Neuronal mechanisms, such as the dysfunction of the sympathetic nerves, are considered as most plausible among a variety of proposed hypotheses [11, 12]. Additionally, somatic mosaicism and microvascular skin homing are proposed as underlying mechanisms of segmental vitiligo. Autoimmune processes are also considered to take part in the pathomechanism of vitiligo; although, it must be noted that segmental vitiligo is rarely associated with various autoimmune diseases as is the case for the non-segmental type [8]. In the presented case, first hypopigmented lesions were observed at the age of 32, and atrophy was 11 years later. This may be considered a late onset for both diseases, potentially determining a mild course of the disease in the case of both syndromes. Parry-Romberg syndrome, as described above, is characterized by a slowly progressing acquired unilateral hemifacial atrophy affecting the subcutaneous tissue, often together with the muscles and the underlying bones. Clinically, the skin of the affected area can be atrophic, dry, or hyperor hypopigmented. In rare cases, skin lesions present a clear demarcation line between the normal and abnormal skin; during the physical examination this has an appearance similar to a scar. More often, as illustrated 
by the presented case, the border between affected and non-affected skin is indistinct. Occasionally, there may be areas of alopecia appearing in patients with hemifacial atrophy, as well as ocular disturbances (most often enopthalmy due to the loss of periorbital fat), epilepsy, facial paresthesia, and other neurological disturbances. In the described patient, trigeminal neuralgia was diagnosed 1 year after the onset of facial atrophy. In more severe cases, often when the disease is noted to start at a younger age than in our patient, deviations of the nose, mouth, and dental midlines (appearing due to tissue atrophy) are observed. Parry-Romberg syndrome and localized scleroderma are considered to be interrelated as both of them have a similar clinicopathological appearance [13-15]. The presence of antinuclear antibodies may suggest that PRS can be a variant of morphea. In our patient, anti-cardiolipin antibodies were elevated; this can occur in $2 \%$ of the healthy population [11]. A similar situation was presented by Bonifati et al. [11], who described a case of linear scleroderma and homolateral segmental vitiligo, where anti-cardiolipin antibodies were elevated. In both cases, no other criteria of the antiphospholipid syndrome were present.

In conclusion, it is difficult to define the correlation between PRS and homolateral segmental vitiligo. Our case is one of the few reported examples of the coexistence of these two disease entities. Further studies are necessary to explain the coexistence of the common pathomechanism in PRS with homolateral vitiligo.

\section{References}

1. Jun JH, Kim HY, Jung HJ, et al. Parry-romberg syndrome with en coup de sabre. Ann Dermatol 2011; 23: 342-7.

2. Kacinski M, Biedron A, Zajac A, Steczkowska M. Diagnostic difficulties of paroxysmal symptoms in a boy with Parry-Romberg syndrome. Neurol Neurochir Pol 2010; 44: 297-303.

3. Budrewicz S, Koszewicz M, Koziorowska-Gawron E, et al. Parry-Romberg syndrome: clinical, electrophysiological and neuroimaging correlations. Neurol Sci 2012; 33: 423-7.

4. Kakisaka Y, So NK, Jones SE, et al. Intractable focal epilepsy contralateral to the side of facial atrophy in Parry-Romberg syndrome. Neurol Sci 2012; 33: 165-8.

5. Miller MT, Spencer MA. Progressive hemifacial atrophy. A natural history study. Trans Am Ophthalmol Soc 1995; 93 : 203-15.

6. Dintiman BJ, Shapiro RS, Hood AF, Guba AM. Parry-Romberg syndrome in association with contralateral Poland syndrome. J Am Acad Dermatol 1990; 22: 371-3.

7. Huggins RH, Janusz CA, Schwartz RA. Vitiligo: a sign of systemic disease. Indian J Dermatol Venereol Leprol 2006; 72 : 68-71.

8. van Geel N, Mollet I, Brochez L, et al. New insights in segmental vitiligo: case report and review of theories. Br J Dermatol 2012; 166: 240-6.

9. van Geel NA, Mollet IG, De Schepper S, et al. First histopathological and immunophenotypic analysis of early dynamic events in a patient with segmental vitiligo associated with halo nevi. Pigment Cell Melanoma Res 2010; 23: 375-84.
10. Osmola-Mańkowska A, Silny W, Dańczak-Pazdrowska A, et al. The sun - our friend or foe? Ann Agric Environ Med 2012; 19: 805-9.

11. Bonifati C, Impara G, Morrone A, et al. Simultaneous occurrence of linear scleroderma and homolateral segmental vitiligo. J Eur Acad Dermatol Venereol 2006; 20: 63-5.

12. Creus L, Sanchez-Regana M, Salleras M, et al. Syndrome de Parry-Romberg associé à un vitiligo segmentaire homolatéral. Ann Dermatol Venereol 1994; 121: 710-1.

13. Bergler-Czop B, Lis-Święty A, Brzezińska-Wcisło L. Progressive facial hemiatrophy without changes in central nervous system and linear scleroderma en coup de sabre with extracerebral tumour - report of two cases [Polish]. Przegl Dermatol 2011; 98: 328-34.

14. Blaszczyk M, Krolicki L, Krasu M, et al. Progressive facial hemiatrophy: central nervous system involvement and relationship with scleroderma en coup de sabre. J Rheumatol 2003; 30: 1997-2004.

15. Tollefson MM, Witman PM. En coup de sabre morphea and Parry-Romberg syndrome: a retrospective review of $54 \mathrm{pa}-$ tients. J Am Acad Dermatol 2007; 56: 257-63. 